\title{
Expression of tissue factor signaling pathway elements correlates with the production of vascular endothelial growth factor and interleukin-8 in human astrocytoma patients
}

\author{
TATIANA C. CARNEIRO-LOBO ${ }^{1 *}$, MARINA T. LIMA $^{2 *}$, ANDRÉA MARIANO-OLIVEIRA $^{1}$, \\ ANGÉLICA DUTRA-OLIVEIRA ${ }^{1}$, SUELI M. OBA-SHINJO ${ }^{3,4}$, SUELY K.N. MARIE ${ }^{3,4}$, \\ MARI C. SOGAYAR ${ }^{2}$ and ROBSON Q. MONTEIRO ${ }^{1}$
}

\author{
${ }^{1}$ Institute of Medical Biochemistry, Federal University of Rio de Janeiro, RJ; ${ }^{2}$ Biochemistry Department, Chemistry Institute, \\ Cell and Molecular Therapy Center (NUCEL), University of São Paulo, SP; ${ }^{3}$ Department of Neurology, \\ School of Medicine, University of São Paulo, SP; ${ }^{4}$ Center of Translational Oncology, \\ The State of São Paulo Cancer Institute (ICESP), SP, Brazil
}

Received September 3, 2013; Accepted October 14, 2013

DOI: $10.3892 /$ or.2013.2880

\begin{abstract}
The expression levels of tissue factor (TF), the clotting initiator protein, have been correlated with angiogenesis and the histological grade of malignancy in glioma patients. The pro-tumor function of TF is linked to a family of $\mathrm{G}$ protein-coupled receptors known as protease-activated receptors (PARs), which may be activated by blood coagulation proteases. Activation of PARs elicits a number of responses, including the expression of vascular endothelial growth factor (VEGF) and interleukin-8 (IL-8). In the present study, we analyzed the expression of TF signaling pathway elements (TF, PAR1 and PAR2) and evaluated their correlation with the expression of downstream products (VEGF and IL-8) in human astrocytoma patients. Quantitative PCR (qPCR) showed a significant increase in TF expression in grade IV (glioblastoma) tumors, which was inversely correlated with the expression of the tumor-suppressor PTEN. Immunohistochemistry and qPCR analyses demonstrated a highly significant elevation in the expression of PAR1, but not PAR2, in tumor samples from high-grade astrocytoma patients. The elevated VEGF expression levels detected in the high-grade astrocytoma samples were positively correlated with TF, PAR1 and PAR2 expression. In addition, IL-8 was significantly increased in glioblastoma patients and positively
\end{abstract}

Correspondence to: Professor Robson Q. Monteiro, Institute of Medical Biochemistry, Federal University of Rio de Janeiro, Av. Carlos Chagas Filho 373, RJ 21941-590, Brazil

E-mail: robsonqm@bioqmed.ufrj.br

*Contributed equally

Key words: blood coagulation, tissue factor, protease-activated receptor, vascular endothelial growth factor, interleukin-8, astrocytoma, glioblastoma correlated with TF and PAR2 expression. Further in vitro assays employing the human glioma cell lines U87-MG and HOG demonstrated that a synthetic peptide PAR2 agonist stimulated VEGF and IL-8 production. Our findings suggest a role for TF signaling pathway elements in astrocytoma progression, particularly in glioblastoma. Therefore, TF/PAR signaling elements may be suitable targets for the development of new therapies for the treatment of aggressive glioma.

\section{Introduction}

Astrocytomas, among gliomas, are the most common type of primary brain malignancies, accounting for $40-50 \%$ of central nervous system tumors. Malignant astrocytomas constitute a spectrum of clinicopathological entities, from low to high-grade malignancies. The World Health Organization (WHO) classifies these tumors into 4 grades according to their histological and anaplastic characteristics, which include: grade I (pilocytic astrocytoma), grade II (low grade astrocytoma), grade III (anaplastic astrocytoma) and grade IV glioblastoma (GBM) (1). GMB is characterized by rapid cell proliferation and a marked propensity to invade and damage the surrounding tissues. In addition, intense angiogenesis is a distinguishing pathological characteristic of GBM relative to lower-grade astrocytomas (2).

Tissue factor (TF), the primary initiator of the coagulation cascade, is a $47-\mathrm{kDa}$ transmembrane protein receptor. Upon disruption of vascular integrity, TF functions as a cofactor for circulating blood factor VIIa, which leads to thrombin activation, platelet aggregation and fibrin deposition (3). TF is constitutively expressed in a cell type specific manner and upregulated in a number of pathological processes $(3,4)$. A strong correlation between TF expression and malignancy grade has been reported for a number of tumors (5). In addition, several studies have implicated the TF-factor VIIa-initiated coagulation pathway in several aspects of tumor biology including cancer progression, cancer-associated thrombosis and metastasis $(6,7)$. 
The occurrence of intratumoral thrombosis in GBM has recently been shown to be an additional distinct feature of grade IV glioma in relation to lower-grade gliomas (8). It has been proposed that intratumoral thrombosis is mainly driven by upregulated TF expression. This increase in TF expression contributes to the establishment of hypoxic areas, further stimulating the production of pro-tumor factors such as vascular endothelial growth factor (VEGF) and interleukin-8 (IL-8) (9,10). In vitro studies demonstrated that hypoxia as well as loss of the PTEN tumor-suppressor upregulate TF gene expression in human GBM cell lines (11). In addition, analysis of human GBM specimens revealed increased TF expression in pseudopalisades, which constitute a dense collection of neoplastic cells that surround a central necrotic focus (11).

In addition to $\mathrm{TF}$, protease-activated receptors (PARs), a family of $\mathrm{G}$ protein-coupled receptors, have been implicated in tumor biology $(6,12)$. PARs comprise a family of receptors (PAR1, PAR2, PAR3 and PAR4) that are uniquely activated by proteolytic cleavage of their extracellular domain (13). This cleavage unmasks a new N-terminus, which serves as a tethered ligand that binds to the second extracellular domain of the protein, resulting in a variety of cellular responses. In particular, PAR1 and PAR2 are overexpressed in a variety of tumor types (14). Several studies have demonstrated a strong correlation between PAR1 and/or PAR2 activation with a number of pro-tumor responses, including primary growth, invasion, metastasis and angiogenesis $(6,12,15)$. It was recently demonstrated that TF-mediated signaling through PAR2 modulates proliferation, migration and invasion of malignant glioma cell lines (16).

In the present study, we analyzed the expression of TF signaling pathway elements (TF, PAR1 and PAR2) as well as downstream products (VEGF and IL-8) in samples from human astrocytoma patients. Our data suggest a role for TF signaling pathway elements in astrocytoma progression, particularly in GBM. Collectively, our results suggest that blood clotting proteins may offer additional strategies for new therapies against aggressive glioma.

\section{Materials and methods}

Reagents. Anti-PAR1 (clone ATAP2), anti-PAR2 (clone SAM11), and anti-actin antibodies were obtained from Santa Cruz Biotechnology, Inc., (Santa Cruz, CA, USA). Secondary antibodies conjugated with biotin and peroxidase-conjugated streptavidin were obtained from Zymed Invitrogen Corp. The PAR1 agonist peptide (PAR1-AP, TFLLR-NH2) and the PAR2 agonist peptide (PAR2-AP, SLIGKL-NH2) were synthesized by Bio-Synthesis Inc.

Tissue samples. Human astrocytomas of different malignant grades were obtained during therapeutical surgical management, as previously described (17). The specimens were immediately snap-frozen in liquid nitrogen upon surgical removal. The astrocytoma specimens were categorized according to the WHO classification system. Then, the tissue samples were analyzed and graded independently by histopathological analysis into the following groups: non-tumor $(n=14)$; pilocytic astrocytoma, grade I $(\mathrm{n}=15)$; low-grade astrocytoma, grade II $(n=15)$; anaplastic astrocytoma, grade III $(n=15)$; or
GBM, grade IV $(\mathrm{n}=30)$. Non-tumor tissue samples, surgically resected from the anterior temporal lobe tissue, were obtained from patients selected for surgical treatment of temporal-lobe epilepsy associated with hippocampus sclerosis (TLE-HS). The present study was performed according to the ethics guidelines approved by the Department of Neurology, School of Medicine, at the University of Sao Paulo and by the Brazilian Health Ministry.

RNA isolation and cDNA synthesis. Total RNA from the astrocytomas and non-tumor samples was isolated with RNeasy Mini kit (Qiagen). Total RNA from the cell cultures was isolated and the mRNA expression levels were determined as previously described (18). The sequence-specific primers were designed using Primer Express (Applied Biosystems) and validated using BLAST and BLAT. Primers used were: TF $(F$, 5'-CAGGCACTACAAATACTGTGG-3' and R, 5'-TGTAGA CTTGATTGACGGGTT-3'); PTEN (F, 5'-CGGTGTCAT AATGTCTTTCAGC-3' and R, 5'-TGAAGGCGTATACAGG AACAAT-3'); PAR1 (F, 5'-GCAGGCCAGAATCAAAAG CAA-3' and R, 5'-CATTTTTCTCCTCATCCTCCC-3'); PAR2 (F, 5'-GCACCATCCAAGGAACCAAT-3' and R, 5'-TGTGC CATCAACCTTACCAATAA-3'); VEGF (F, 5'-AGTGGT GAAGTTCATGGATGT-3' and R, 5'-GCACACAGGAT GGCTTGAAGA-3'); IL-8 (F, 5'-CTGGACCCCAAGGAAA ACTG-3' and R, 5'-TGTGCCATCAACCTTACCAATAA-3'); GAPDH (F, 5'-ACCCACTCCTCCACCTTTGA-3' and R, 5'-ACCGAGCCCATTTCATTTCTG-3') and HBMS (F, 5'-TGGACCTGGTTGTTCACTCCTT-3' and R, 5'-CAACAG CATCATGAGGGTTTTC-3'). A normalization value was generated employing the geNorm program, which selected GAPDH and HBMS as housekeeping genes. The relative expression levels were estimated utilizing a previously described method, using the mean of control non-tumor samples as calibrator (19).

Immunohistochemistry. Tissue staining was performed on paraffin-embedded sections ( $4 \mu \mathrm{m}$ thick), which were incubated overnight following heat antigen retrieval at $122^{\circ} \mathrm{C}$ for 3 min using an electric pressure cooker (BioCase Medical, USA) with the primary antibodies anti-PAR1 or anti-PAR2 at 1:400 dilution. The sections were further examined using a NovoLink kit (Newcastle upon Tyne, UK) with 3,3'-diaminobenzidine as the chromogen and counterstained with Harris hematoxylin. Optimization using positive controls suggested by the manufacturer of each antibody was performed in order to obtain optimal dilution. The negative control sections were obtained by omitting the primary antibody in each staining batch. The slides were independently analyzed by 2 observers (SKNM and SMOS). The immunoreactivities of PAR1 and PAR2 were determined semi-quantitatively in 5 cases of each grade of malignancy and non-tumor brain tissue, considering both intensity of staining and percentage of cells. Intensity of staining was applied as follows: 0 (negative); 1 (weak), 2 (moderate) and 3 (strong). The percentage of stained cells was determined according to the following scale: negative (no cells stained), low (1-25\%), moderate (26-50\%), high (51-75\%), and strong intensity (>75\%). The total score for each case was calculated as the percentage vs. intensity. 
Cell culture. The human GBM cell line U87-MG and oligodendroglioma cell line HOG were cultured as previously described (18). Adult primary human astrocytes were isolated from non-tumor tissues collected as described above and further cultured as previously described (20). Primary cultures were used until the third passage in the present study. For the experiments, the cells were maintained in DMEM-F12 (Gibco-BRL) supplemented with 10\% FBS (Cultilab, Brazil), $60 \mathrm{mg} / \mathrm{l}$ penicillin, $100 \mathrm{mg} / \mathrm{l}$ streptomycin and $1.2 \mathrm{~g} / \mathrm{l}$ sodium bicarbonate in culture flasks. The cells were incubated in 5\% $\mathrm{CO}_{2}$ at $37^{\circ} \mathrm{C}$. The subconfluent cultures were washed twice with PBS, and the cells were detached with Hank's solution containing $10 \mathrm{mM}$ HEPES and $0.2 \mathrm{mM}$ EDTA.

Western blotting. Adult primary human astrocytes, U87-MG and HOG cells were seeded at $5 \times 10^{5}$ cells/well in 6 -well plates for the quantitative analysis of PAR1 and PAR2, as previously described (21) the cells were washed with phosphate-buffered saline and lysed in cold buffer containing a phosphatase inhibitor cocktail. The cell lysates $(20 \mu \mathrm{l})$ were separated by SDS-polyacrylamide gel electrophoresis (SDS-PAGE, 10\%). The proteins were transferred onto polyvinylidene fluoride (PVDF) membranes (Millipore) and further blocked with Trisbuffered saline (TBS) containing 5\% BSA and 0.1\% Tween-20 for $1 \mathrm{~h}$ at room temperature. Then, the membranes were probed with the primary antibodies overnight at $4^{\circ} \mathrm{C}$. The membranes were washed 3 times with TBS/Tween before addition of the secondary antibody for $1 \mathrm{~h}$ at room temperature, and further washed and probed with peroxidase-conjugated streptavidin for $1 \mathrm{~h}$ at room temperature. Immunodetection was carried out with a chemiluminescent method using a Western Lightning ECL kit (Amersham Pharmacia Biotech).

Immunoassay for IL-8 and VEGF. U87-MG and HOG cells were seeded at $5 \times 10^{6}$ cells/well in 6 -well plates. The cells were serum-starved for 30 min prior to stimulation with PAR1-AP or PAR2-AP for $8 \mathrm{~h}$ at $37^{\circ} \mathrm{C}$. IL-8 and VEGF protein secretion into the cell supernatants was measured using human ELISA kits from PeproTech, Inc. (Rocky Hill, NJ, USA), according to the manufacturer's instructions.

Statistical analysis. All statistical analyses were performed using the GraphPad Prism program. The gene expression assays were analyzed using the Mann-Whitney test (Student's t- and non-parametric test). Immunohistochemistry was analyzed by one-way analysis of variance (ANOVA) complemented by the Bonferroni post-hoc test. The Spearman's correlation test was used for correlation analysis. Immunoassay for IL-8 and VEGF were analyzed by unpaired t-test. The differences were considered to be statistically significant at $\mathrm{p}<0.05$.

\section{Results}

Expression of TF and PTEN are inversely correlated in human astrocytoma patients. Previous studies demonstrated that TF expression correlates with the histological grade of malignancy in glioma patients $(22,23)$. In the present study, we analyzed TF expression in 89 samples which included non-tumor tissues and astrocytoma patient specimens classified into different grades. Analysis by qPCR demonstrated
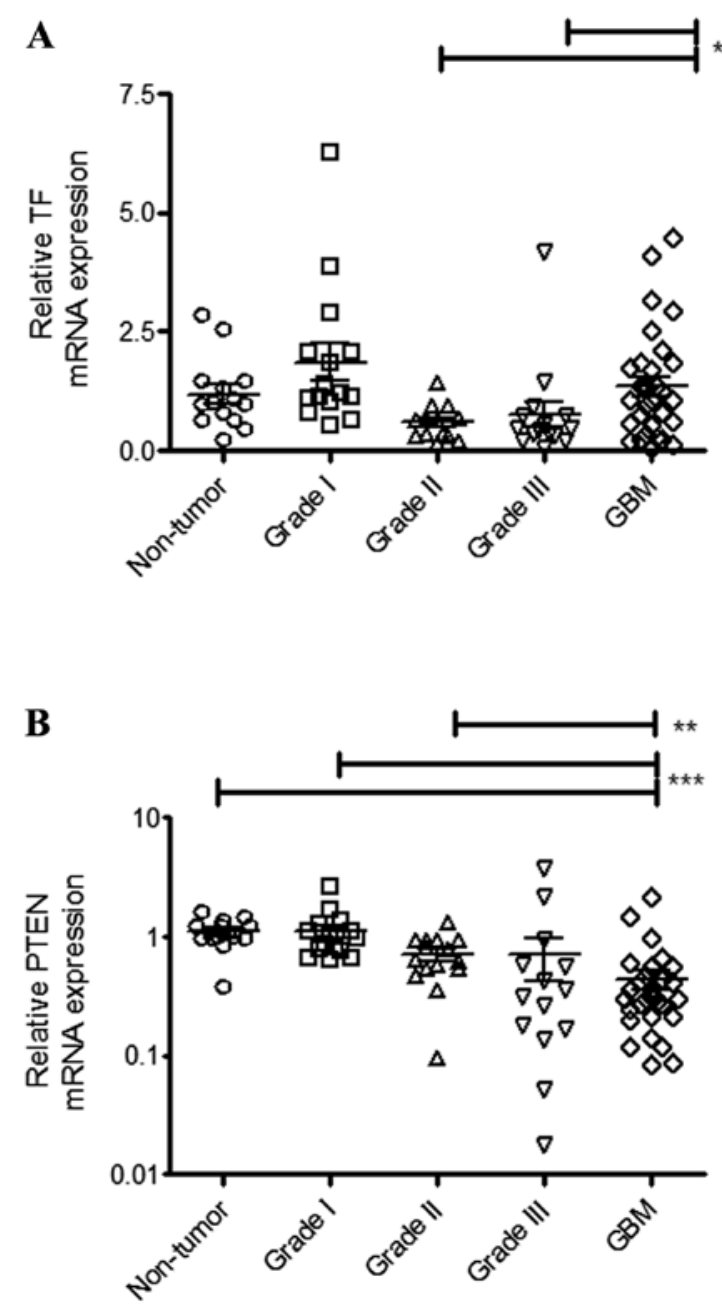

Figure 1. Analysis of TF and PTEN RNA expression in human astrocytomas of different grades of malignancy. (A) TF and (B) PTEN gene expression levels evaluated by qPCR were normalized to GAPDH and HBMS as housekeeping genes. Mean value $( \pm$ SEM) for each group is indicated by a horizontal line. Statistical significance was assessed by the Mann-Whitney test. ${ }^{*} \mathrm{p}<0.05,{ }^{* *} \mathrm{p}<0.01,{ }^{* * *} \mathrm{p}<0.001$. TF, tissue factor.

that $\mathrm{TF}$ expression was positive in all the samples (Fig. 1A). However, as shown in Fig. 1A, a significantly elevated TF expression was only observed in the GBM samples relative to grade II ( $\mathrm{p}=0.0356)$ and grade III $(\mathrm{p}=0.0312)$ astrocytoma patients.

In vitro studies suggested that upregulation of TF in highgrade gliomas is associated with loss of the tumor-suppressor PTEN (11). Consistent with this hypothesis, Fig. 1B shows that PTEN expression levels were inversely correlated with the malignancy in astrocytoma patients. We found a significant correlation between PTEN and TF mRNA expression levels in GBM samples (Table I).

Expression of PAR1, but not PAR2, is correlated with the malignancy in human astrocytoma patients. The pro-tumor role of TF has been correlated with its ability to generate thrombin and indirectly promote the activation of the $\mathrm{G}$ protein-coupled receptor PAR1 $(5,6)$. Furthermore, elevated expression levels of PAR1 have been demonstrated in studies employing tumor cell lines and patient specimens (14). 

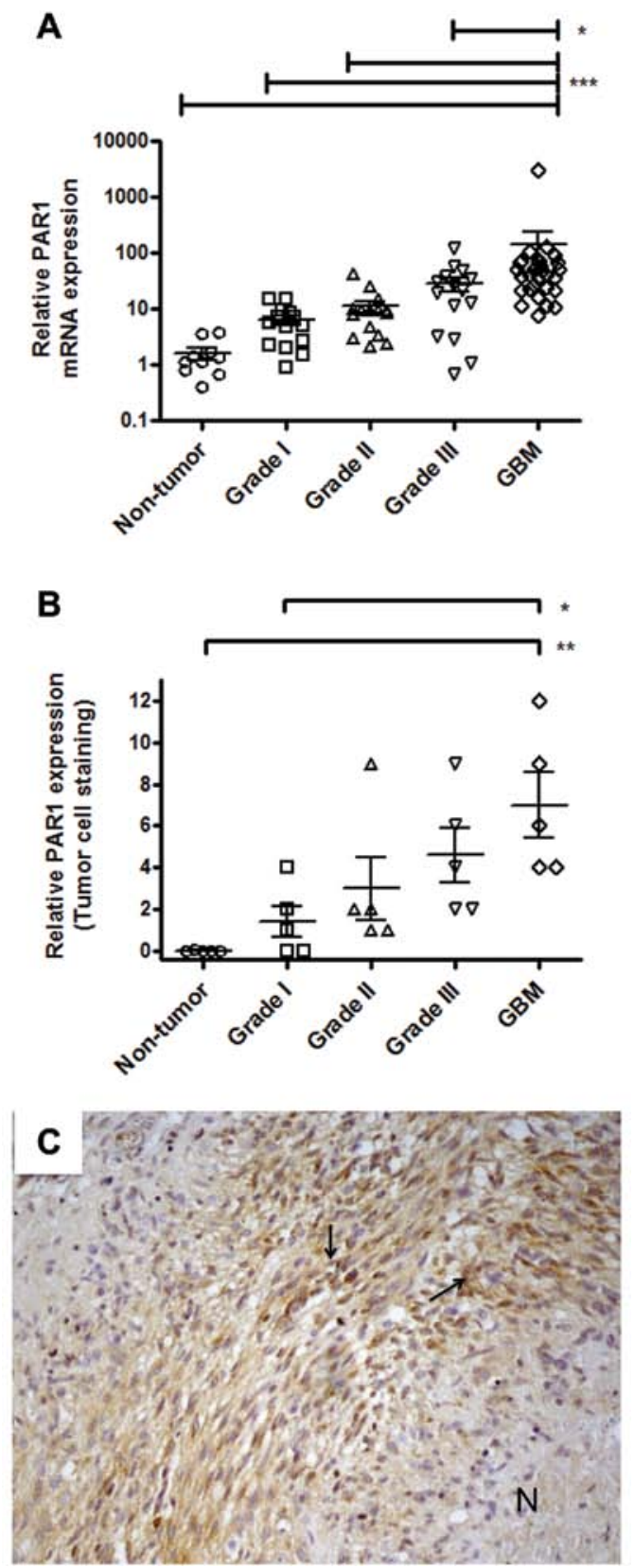

Figure 2. Analysis of PAR1 expression in human non-tumor tissue and astrocytoma samples of different grades of malignancy. (A) PAR1 mRNA expression levels evaluated by qPCR were normalized to GAPDH and HBMS as housekeeping genes. Mean value ( \pm SEM) for each group is indicated by a horizontal line. Statistical significance was assessed by the Mann-Whitney test. ${ }^{*} \mathrm{p}<0.05,{ }^{* * *} \mathrm{p}<0.001$. (B) Immunohistochemical staining for PAR1 in nontumor and astrocytoma surgical specimens. Mean value ( \pm SEM) for each group is indicated by a horizontal line. Statistical significance was assessed by one-way ANOVA followed by post-hoc Bonferroni test. ${ }^{*} \mathrm{p}<0.05,{ }^{* *} \mathrm{p}<0.01$ (C) Representative immunostaining demonstrates that PAR1 expression is present within cells at the inner aspect of hypoxic pseudopalisades (arrows) surrounding necrosis (N) of human GBM specimens (original magnification, $\mathrm{x} 200)$. The staining procedure is described in the Materials and methods section. GBM, glioblastoma.

Analysis by qPCR showed that PAR1 expression levels were positively correlated with malignancy in astrocytoma patients, as shown in Fig. 2A. PAR1 mRNA expression levels were significantly more abundant in GBM when compared to the non-tumor tissue $(\mathrm{p}<0.0001)$ and to lower grade astrocytoma samples $(\mathrm{p}<0.0001$, relative to grade I; $p<0.0001$,
A

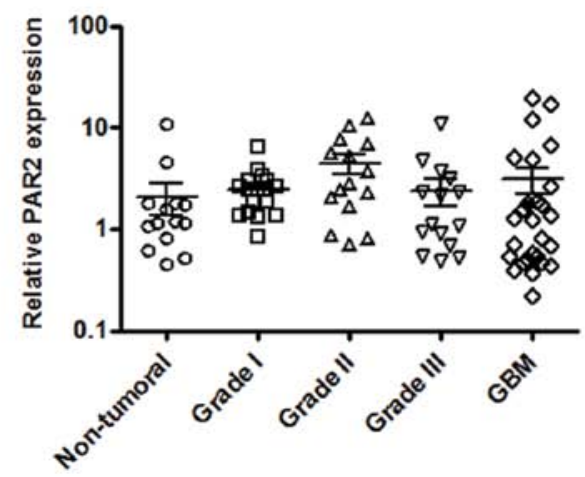

B
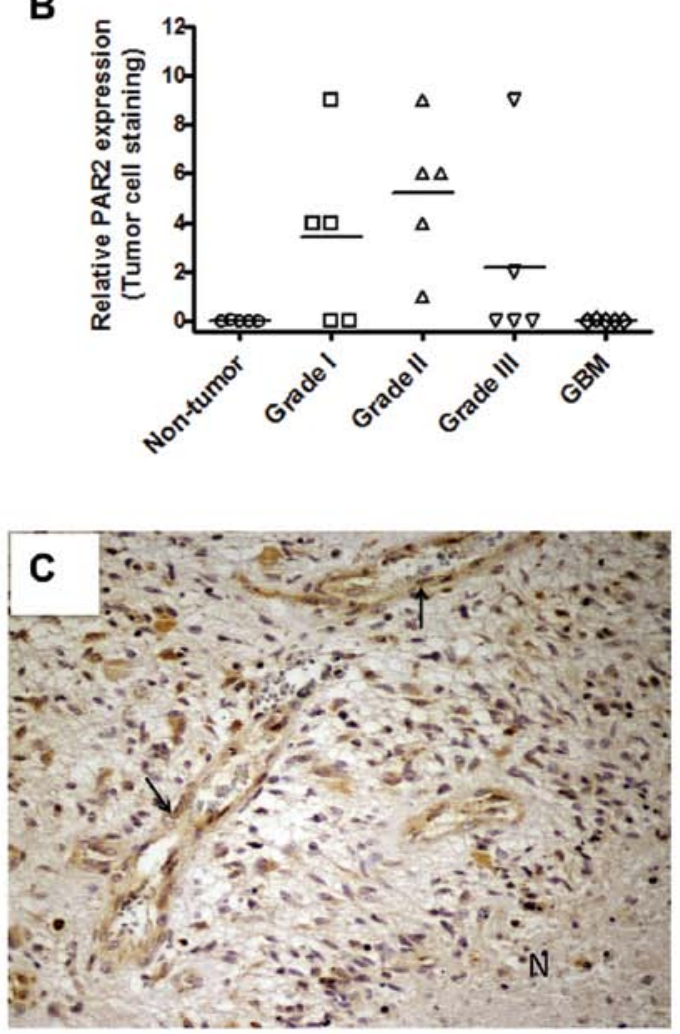

Figure 3. Analysis of PAR2 expression in human non-tumor tissue and astrocytoma samples of different grades of malignancy. (A) PAR2 mRNA expression levels evaluated by qPCR were normalized to GAPDH and HBMS as housekeeping genes. Mean value $( \pm$ SEM) for each group is indicated by a horizontal line. Statistical significance was evaluated by the Mann-Whitney test. (B) Immunohistochemical staining analysis for PAR2 in non-tumor and astrocytoma surgical specimens. Statistical significance was evaluated by one-way ANOVA followed by post-hoc Bonferroni test. (C) Representative immunostaining demonstrates that PAR2 expression is present within cells at the inner aspect of hypoxic pseudopalisades (arrows) surrounding necrosis $(\mathrm{N})$ of human GBM specimens (original magnification, $\mathrm{x} 200$ ). The staining procedure is described in the Materials and methods section. GBM, glioblastoma.

relative to grade II; $\mathrm{p}=0.0146$ relative to grade III). These results were further confirmed by immunohistochemistry analyses, as shown in Fig. 2B. PAR1 staining in the tumorassociated endothelium was significantly elevated in GBM when compared to non-tumor tissue $(\mathrm{p}=0.001)$ and samples from lower-grade astrocytoma (data not shown). 
Table I. Relationship between the expression levels of TF, PAR1, PAR2 and analyzed genes in GBM.

\begin{tabular}{|c|c|c|c|c|c|c|}
\hline & \multicolumn{2}{|c|}{$T F$} & \multicolumn{2}{|c|}{ PARI } & \multicolumn{2}{|c|}{ PAR2 } \\
\hline & $\mathrm{P}$ & $\mathrm{r}$ & $\mathrm{P}$ & $\mathrm{r}$ & $\mathrm{P}$ & $\mathrm{r}$ \\
\hline PTEN & 0.0004 & 0.3979 & NS & -0.1748 & 0.0002 & 0.4246 \\
\hline VEGF & 0.0018 & 0.3550 & $<0.0001$ & 0.5939 & 0.0115 & -0.3003 \\
\hline IL-8 & $<0.0001$ & 0.9997 & NS & 0.1572 & 0.1563 & 0.1688 \\
\hline
\end{tabular}

P, Spearman's rank test; r, correlation coefficient, Spearman's rank test; NS, non-significant; TF, tissue factor; GBM, glioblastoma; VEGF, vascular endothelial growth factor; IL-8, interleukin-8.

In addition to PAR1, PAR2 has been shown to be overexpressed in several types of cancer (14). Therefore, we further employed qPCR and immunohistochemistry for analysis of PAR2 expression in tissue samples. Fig. 3A shows that mRNA encoding PAR 2 was detected in all the samples analyzed, but no significant difference was observed between the non-tumor tissue and astrocytoma samples. These observations were further confirmed by immunohistochemistry, as shown in Fig. 3B. The immunohistochemistry results were somewhat heterogeneous and some astrocytoma samples demonstrated high antigen reactivity either in tumor cells (Fig. 3B) or in tumor-associated endothelium (data not shown).

A stronger positive staining was observed around the perinecrotic area mainly in pseudopalisading cells for either PAR1 (Fig. 2C) or PAR2 (Fig. 3C), although PAR2 staining was more intense than that observed in PAR1.

Expression of VEGF and IL-8 correlates with TF signaling pathway elements. A key event that accompanies astrocytoma progression is the upregulation of VEGF levels. Intense angiogenesis is a pathological hallmark of GBM relative to lower-grade gliomas (2). GBM is a highly vascularized malignant tumor and there is strong evidence that VEGF plays a key role in this process (24). In this context, Fig. 4A shows that VEGF expression is significantly upregulated in GBM relative to non-tumor tissues $(\mathrm{p}<0.0001)$ and grade $\mathrm{I}(\mathrm{p}<0.0001)$, grade II $(\mathrm{p}<0.0001)$ and grade III $(\mathrm{p}<0.0001)$ astrocytomas.

In addition to VEGF, the chemokine IL- 8 has been recognized as an important factor in astrocytoma progression and GBM angiogenesis $(25,26)$. Accordingly, our data demonstrate that GBM samples exhibit increased expression of IL-8 when compared to grade II $(\mathrm{p}=0.0098)$ and grade III $(p=0.0420)$ astrocytomas (Fig. 4B). Furthermore, we evaluated the correlation between the TF mRNA expression levels of the angiogenic factors. We found a significant correlation between TF and VEGF expression, as well as between TF and IL-8 expression (Table I). Analysis of the correlation between the mRNA expression levels of PAR1 or PAR2 and the angiogenic factors in GBM samples showed a significant correlation between PAR1 and VEGF expression (Table I). There was no significant correlation between PAR1 and IL-8 expression. Furthermore, PAR2 expression was correlated with VEGF, but not with IL-8 (Table I).
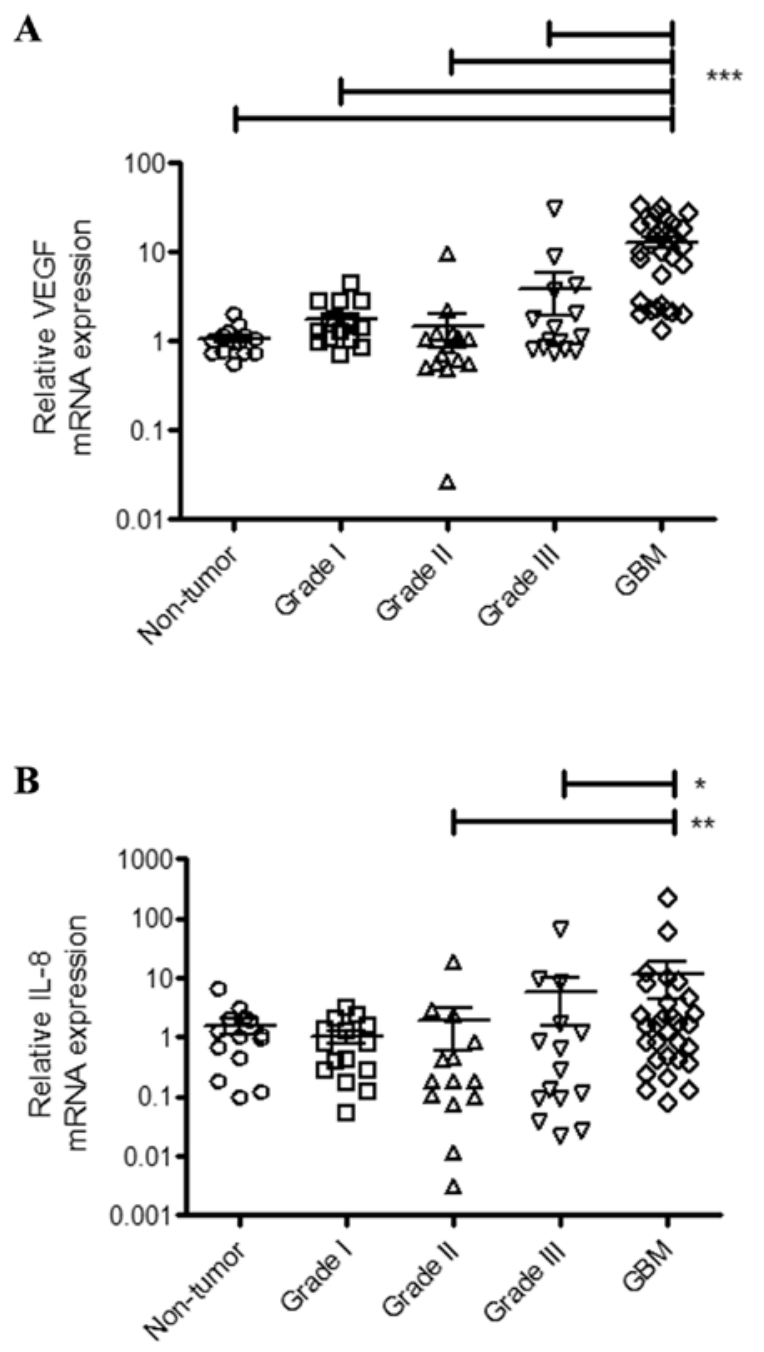

Figure 4. Analysis of VEGF and IL-8 mRNA expression in human non-tumor tissue and astrocytoma samples of different grades of malignancy. (A) VEGF and (B) IL-8 gene expression levels evaluated by qPCR were normalized to GAPDH and HBMS as housekeeping genes. Mean value ( \pm SEM) for each group is indicated by a horizontal line. Statistical significance was assessed by the Mann-Whitney test. ${ }^{*} \mathrm{p}<0.05,{ }^{* *} \mathrm{p}<0.01,{ }^{* * *} \mathrm{p}<0.001$. VEGF, vascular endothelial growth factor; IL-8, interleukin-8, GBM, glioblastoma.

A PAR2 agonist stimulates VEGF and IL-8 production in vitro. Several studies demonstrated that PAR1 and PAR2 are constitutively expressed in a variety of tumor cell lines. Accordingly, Fig. 5A compares the expression of PAR1 and PAR2 in primary astrocytes to the human GBM cell line U87-MG and the human oligodendroglioma cell line HOG. Western blot analysis showed no detectable levels of PAR1 or PAR2 in the astrocytes, while both the tumor cell lines constitutively expressed PAR1 and PAR2. Both receptors have been implicated in VEGF and IL- 8 production by tumor cells $(15,21,27)$. To determine the effect of PAR1 and PAR2 activation on VEGF and IL-8 production in the U87-MG and HOG cell lines, we employed synthetic agonist peptides and further quantified VEGF and IL-8 in the conditioned medium. Fig. 5B shows that activation of PAR2, but not PAR1, increased VEGF production in both cell lines. In addition, the PAR2 agonist peptide induced IL-8 production in U87-MG, but not in HOG, cells. 


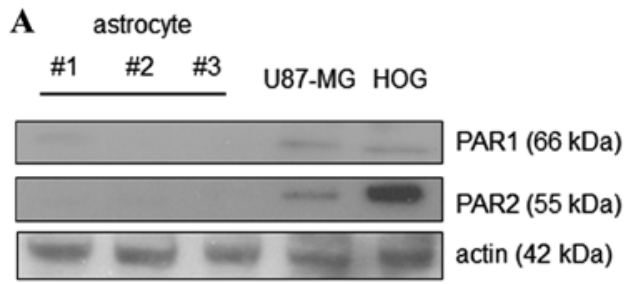

B

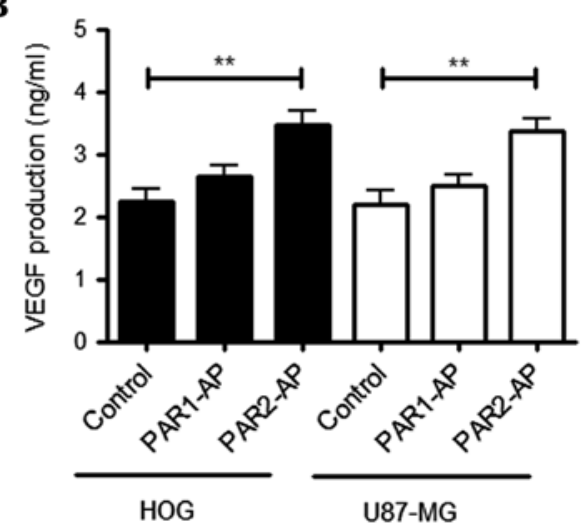

C

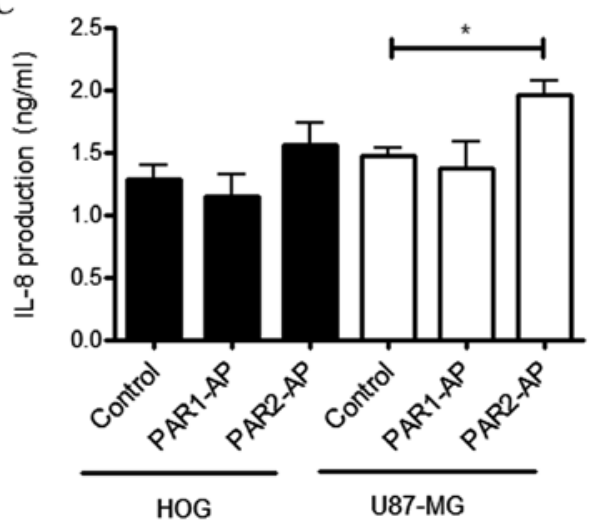

Figure 5. PAR2 activation induces VEGF and IL-8 secretion in human glioma cell lines. (A) Western blotting using PAR1 and PAR2 antibodies. Primary astrocytes, U87-MG and HOG cell lines were serum-starved overnight. Adult primary human astrocytes were isolated from non-tumor tissues and further cultured following procedures described in the Materials and methods section. Total levels of PAR1, PAR2 and actin were determined by immunoblotting as described in the Materials and methods section. (B and C) VEGF and IL-8 levels were quantified in the conditioned media after treatment of the indicated cell lines with PAR1-AP $(100 \mu \mathrm{M})$ or PAR2-AP $(100 \mu \mathrm{M})$ for $20 \mathrm{~h}$, in serum-free conditions. VEGF and IL-8 secretion into cell supernatants were quantified as described in the Materials and methods section. The data are shown as the means \pm SD of 3 independent experiments. Statistical significance was assessed by an unpaired t-test. "p $<0.05,{ }^{* *} \mathrm{p}<0.01$. VEGF, vascular endothelial growth factor; IL-8, interleukin-8.

\section{Discussion}

A marked shift in biological behavior occurs following the transition from low- to high-grade astrocytoma. These changes include the acquisition of proliferative and invasive phenotypes, which account for the extensive damage in normal brain tissue. Intense angiogenesis is another pathological hallmark of high-grade glioma. Indeed, GBM is one of the most highly vascularized malignant tumors. Despite significant advances in diagnostics and characterization of molecular markers associated with aggressive astrocytoma, the search for new targets remains a challenge (28). The present study provides evidence that the signaling pathways triggered by the clotting initiator protein TF may play a role in GBM progression. Expression of TF, as well as PAR1 and PAR2, correlated with increased levels of VEGF and IL-8, which are well recognized factors in astrocytoma progression and GBM aggressiveness.

Intratumoral thrombosis has been documented as an additional distinguishing pathological feature in GBM relative to lower grade astrocytoma (8). According to our results, this may be explained by the significant elevation of TF expression in GBM. Different mechanisms have been proposed for the increased expression of TF in high-grade glioma including hypoxia and oncogenic events $(10,11,27)$. In vitro studies employing tumor cell lines have demonstrated that loss of the tumor-suppressor PTEN is directly correlated with upregulation of TF (11). In accordance with these observations, we demonstrated that decreased PTEN mRNA levels correlate with increased TF expression in astrocytoma patients.

Studies employing tumor cell lines demonstrated that simultaneous TF expression and phosphatidylserine exposure contribute to massive generation of clotting enzymes $(29,30)$, which may account for the activation of signaling pathways elicited by cleavage of PARs. PAR1 and PAR2 activation have been associated with transcriptional programs regulating increased expression of angiogenic molecules $(6,15)$. Our results demonstrate that PAR1 expression is correlated with malignancy and is highly expressed in high-grade astrocytoma. This is in agreement with the immunohistochemistry data from a study performed by Zhang et al (31), which also demonstrated that PAR1 expression levels are an independent prognostic factor in GBM patients. Notably, the authors reported the significant upregulation of metalloprotease-1 (MMP1) in high-grade glioma. MMP-1 has been described as a possible PAR1 activator and may possibly elicit PAR1 pro-tumor effects through a TF-independent route (32). PAR1 supports oncogenic transformation and angiogenesis by upregulating VEGF in vitro and in vivo (33). In this context, elevated levels of PAR1 in GBM may directly contribute to the angiogenic switch during astrocytoma progression. This hypothesis is reinforced by the observation that thrombin, which activates PAR1, upregulates VEGF production in vitro and co-localizes with VEGF in vivo in a rat glioma model (34).

TF-dependent activation of PAR2 has recently been implicated in increased proliferation, migration and invasion of GBM cell lines (16). Antibodies that specifically target TF-dependent signaling without affecting TF procoagulant activity diminish these pro-tumor properties. Inhibition of proliferation, migration and invasion of GBM cell lines has also been attained by independently silencing TF or PAR2. Studies employing a spontaneous mammary tumor model in mice demonstrated that the TF/PAR2 signaling axis is coupled to angiogenesis in breast cancer $(35,36)$. Thus, PAR2-deficient mice have delayed mammary tumor progression, as well as decreased angiogenesis in the initial phase of tumor development. Svensson et al demonstrated that PAR2 activation in endothelial cells may be triggered by TF-bearing microvesicles derived from GBM cells (37). This phenomenon is observed particularly under hypoxic conditions and is consistent with 
the observation that TF is more highly expressed proximal to the necrotic areas in GBM samples (11). Recently, Harter et al demonstrated that PAR2 is heterogeneously overexpressed in GBM (38). These findings may explain our observation that analyses of PAR2 expression by qPCR or immunohistochemistry show no differences between GBM and lower-grade gliomas as well as between GBM and non-tumor samples.

Targeting the blood clotting cascade may be a feasible therapeutic approach for treatment of GBM (39). In this regard, argatroban, a specific thrombin inhibitor, has been shown to reduce the in vivo growth of rat GBM. However, argatroban treatment in this model displayed only a modest improvement in survival (40). It was demonstrated that Ixolaris, a TF inhibitor that blocks PAR2 signaling in vitro (41), blocks the in vivo growth of human GBM (U87-MG) cells in a xenograft model (18). This phenomenon was accompanied by a significant decrease in VEGF expression as well as diminished tumor angiogenesis. Additionally, Harter et al demonstrated that a monoclonal anti-TF antibody reduces tumor cell invasiveness in a xenograft model (38).

Collectively, our results strongly suggest that signaling pathway elements comprising TF, PAR1 and PAR2 are connected to the upregulation of VEGF and IL-8 expression in high-grade glioma. Therefore, these proteins may offer additional targets for the development of new therapies to treat aggressive glioma.

\section{Acknowledgements}

The authors thank Zizi de Mendonça for the technical assistance. The present study was supported by the Brazilian National Council for Scientific and Technological Development (CNPq), the State of Rio de Janeiro Research Foundation (FAPERJ), the State of São Paulo Research Foundation (FAPESP), and the Brazilian Cancer Foundation.

\section{References}

1. Louis DN, Ohgaki H, Wiestler OD, Cavenee WK, Burger PC, Jouvet A, Scheithauer BW and Kleihues P: The 2007 WHO classification of tumours of the central nervous system. Acta Neuropathol 114: 97-109, 2007.

2. Wen PY and Kesari S: Malignant gliomas in adults. N Engl J Med 359: 492-507, 2008.

3. Williams JC and Mackman N: Tissue factor in health and disease. Front Biosci 1: 358-372, 2012.

4. Francischetti IM, Seydel KB and Monteiro RQ: Blood coagulation, inflammation, and malaria. Microcirculation 15: 81-107, 2008.

5. Rak J, Milsom C, Magnus $\mathrm{N}$ and $\mathrm{Yu} \mathrm{J}$ : Tissue factor in tumour progression. Best Pract Res Clin Haematol 22: 71-83, 2009.

6. Ruf W, Disse J, Carneiro-Lobo TC, Yokota N and Schaffner F: Tissue factor and cell signalling in cancer progression and thrombosis. J Thromb Haemost 9 (Suppl 1): 306-315, 2011.

7. Lima LG and Monteiro RQ: Activation of blood coagulation in cancer: implications for tumor progression. Biosci Rep 33: e00064, 2013.

8. Tehrani M, Friedman TM, Olson JJ and Brat DJ: Intravascular thrombosis in central nervous system malignancies: a potential role in astrocy toma progression to glioblastoma. Brain Pathol 18: 164-171, 2008

9. Brat DJ and Van Meir EG: Vaso-occlusive and prothrombotic mechanisms associated with tumor hypoxia, necrosis, and accelerated growth in glioblastoma. Lab Invest 84: 397-405, 2004

10. Anand $M$ and Brat DJ: Oncogenic regulation of tissue factor and thrombosis in cancer. Thromb Res 129 (Suppl 1): S46-S49, 2012 .
11. Rong Y, Post DE, Pieper RO, Durden DL, Van Meir EG and Brat DJ: PTEN and hypoxia regulate tissue factor expression and plasma coagulation by glioblastoma. Cancer Res 65: 1406-1413, 2005.

12. Kasthuri RS, Taubman MB and Mackman N: Role of tissue factor in cancer. J Clin Oncol 27: 4834-4838, 2009.

13. Coughlin SR: Protease-activated receptors in hemostasis, thrombosis and vascular biology. J Thromb Haemos 3: 1800-1814, 2005.

14. Elste AP and Petersen I: Expression of proteinase-activated receptor 1-4 (PAR 1-4) in human cancer. J Mol Histol 41: 89-99, 2010.

15. Albrektsen T, Sørensen BB, Hjort $\varnothing$ GM, Fleckner J, Rao LV and Petersen LC: Transcriptional program induced by factor VIIa-tissue factor, PAR1 and PAR2 in MDA-MB-231 cells. J Thromb Haemost 5: 1588-1597, 2007.

16. Gessler F, Voss V, Dützmann S, Seifert V, Gerlach R and Kögel D: Inhibition of tissue factor/protease-activated receptor-2 signaling limits proliferation, migration and invasion of malignant glioma cells. Neuroscience 165: 1312-1322, 2010.

17. Oba-Shinjo SM, Bengtson MH, Winnischofer SM, Colin C, Vedoy CG, de Mendonça Z, Marie SK and Sogayar MC: Identification of novel differentially expressed genes in human astrocytomas by cDNA representational difference analysis. Brain Res Mol Brain Res 140: 25-33, 2005.

18. Carneiro-Lobo TC, Konig S, Machado DE, Nasciutti LE, Forni MF, Francischetti IM, Sogayar MC and Monteiro RQ: Ixolaris, a tissue factor inhibitor, blocks primary tumor growth and angiogenesis in a glioblastoma model. J Thromb Haemost 7: 1855-1864, 2009.

19. Livak KJ and Schmittgen TD: Analysis of relative gene expression data using real-time quantitative PCR and the $2^{-\Delta \Delta C t}$ Methods 25: 402-408, 2001.

20. De Groot CJ, Langeveld CH, Jongenelen CA, Montagne L, Van Der Valk P and Dijkstra CD: Establishment of human adult astrocyte cultures derived from postmortem multiple sclerosis and control brain and spinal cord regions: immunophenotypical and functional characterization. J Neurosci Res 49: 342-354, 1997.

21. Dutra-Oliveira A, Monteiro RQ and Mariano-Oliveira A: Protease-activated receptor-2 (PAR2) mediates VEGF production through the ERK1/2 pathway in human glioblastoma cell lines. Biochem Biophys Res Commun 421: 221-227, 2012.

22. Hamada K, Kuratsu J, Saitoh Y, Takeshima H, Nishi T and Ushio Y: Expression of tissue factor correlates with grade of malignancy in human glioma. Cancer 77: 1877-1883, 1996.

23. Guan M, Jin J, Su B, Liu WW and Lu Y: Tissue factor expression and angiogenesis in human glioma. Clin Biochem 35: 321-325, 2002.

24. Plate KH, Breier G, Weich HA and Risau W: Vascular endothelial growth factor is a potential tumour angiogenesis factor in human gliomas in vivo. Nature 359: 845-848, 1992.

25. Koch AE, Polverini PJ, Kunkel SL, Harlow LA, DiPietro LA Elner VM, Elner SG and Strieter RM: Interleukin-8 as a macrophage-derived mediator of angiogenesis. Science 258: 1798-1801, 1992.

26. Brat DJ, Bellail AV and Van Meir EG: The role of interleukin-8 and its receptors in gliomagenesis and tumoral angiogenesis. Neuro Oncol 7: 122-133, 2005.

27. Magnus N, Garnier D and Rak J: Oncogenic epidermal growth factor receptor up-regulates multiple elements of the tissue factor signaling pathway in human glioma cells. Blood 116: 815-818, 2010.

28. Lima FR, Kahn SA, Soletti RC, Biasoli D, Alves T, da Fonseca AC, Garcia C, Romão J, Brito L, Holanda-Afonso R, Faria J, Borges H and Moura-Neto V: Glioblastoma: therapeutic challenges, what lies ahead. Biochim Biophys Acta 1826: 338-349, 2012.

29. Fernandes RS, Kirszberg C, Rumjanek VM and Monteiro RQ: On the molecular mechanisms for the highly procoagulant pattern of C6 glioma cells. J Thromb Haemost 4: 1546-1552, 2006.

30. Kirszberg C, Lima LG, Da Silva de Oliveira A, Pickering W, Gray E, Barrowcliffe TW, Rumjanek VM and Monteiro RQ: Simultaneous tissue factor expression and phosphatidylserine exposure account for the highly procoagulant pattern of melanoma cell lines. Melanoma Res 19: 301-308, 2009.

31. Zhang Y, Zhan H, Xu W, Yuan Z, Lu P, Zhan L and Li Q: Upregulation of matrix metalloproteinase-1 and proteinaseactivated receptor-1 promotes the progression of human gliomas. Pathol Res Pract 207: 24-29, 2011.

32. Boire A, Covic L, Agarwal A, Jacques S, Sherifi S and Kuliopulos A: PAR1 is a matrix metalloprotease-1 receptor that promotes invasion and tumorigenesis of breast cancer cells. Cell 120: 303-313, 2005. 
33. Yin YJ, Salah Z, Maoz M, Even Ram SC, Ochayon S, Neufeld G, Katzav S and Bar-Shavit R: Oncogenic transformation induces tumor angiogenesis: a role for PAR1 activation. FASEB J 17: 163-174, 2003.

34. Xu Y, Gu Y, Keep RF, Heth J, Muraszko KM, Xi G and Hua Y: Thrombin up-regulates vascular endothelial growth factor in experimental gliomas. Neurol Res 31: 759-765, 2009.

35. Versteeg HH, Schaffner F, Kerver M, Ellies LG, AndradeGordon P, Mueller BM and Ruf W: Protease-activated receptor (PAR) 2, but not PAR1, signaling promotes the development of mammary adenocarcinoma in polyoma middle $\mathrm{T}$ mice. Cancer Res 68: 7219-7227, 2008.

36. Schaffner F, Versteeg HH, Schillert A, Yokota N, Petersen LC, Mueller BM and Ruf W: Cooperation of tissue factor cytoplasmic domain and PAR2 signaling in breast cancer development. Blood 116: 6106-6113, 2010.

37. Svensson KJ, Kucharzewska P, Christianson HC, Sköld S, Löfstedt T, Johansson MC, Mörgelin M, Bengzon J, Ruf W and Belting M: Hypoxia triggers a proangiogenic pathway involving cancer cell microvesicles and PAR-2-mediated heparin-binding EGF signaling in endothelial cells. Proc Natl Acad Sci USA 108: 13147-13152, 2011.
38. Harter PN, Dützmann S, Drott U, Zachskorn C, Hattingen E, Capper D, Gessler F, Senft C, Seifert V, Plate KH, Kögel D and Mittelbronn M: Anti-tissue factor (TF9-10H10) treatment reduces tumor cell invasiveness in a novel migratory glioma model. Neuropathology 33: 515-525, 2013.

39. Ornstein DL, Meehan KR and Zacharski LR: The coagulation system as a target for the treatment of human gliomas. Semin Thromb Hemost 28: 19-28, 2002.

40. Hua Y, Tang L, Keep RF, Schallert T, Fewel ME, Muraszko KM, Hoff JT and Xi G: The role of thrombin in gliomas. J Thromb Haemost 3: 1917-1923, 2005.

41. Carneiro-Lobo TC, Schaffner F, Disse J, Ostergaard H, Francischetti IM, Monteiro RQ and Ruf W: The tick-derived inhibitor Ixolaris prevents tissue factor signaling on tumor cells. J Thromb Haemost 10: 1849-1858, 2012. 